\title{
RELEVANCIA DE LOS COLIBRÍES (AVES, TROCHILIDAE) COMO COMPLEJO DE ESPECIES BIOCULTURALES. EL COLIBRÍ Y EL ANDAR DEL TIEMPO NASA - E'C A'TE (TIEMPOS DE LA ESMERALDA)
}

\author{
RELEVANCE OF HUMMINGBIRDS (AVES, TROCHILIDAE) AS A COMPLEX OF \\ BIOCULTURAL SPECIES. THE COLIBRÍ AND THE WALK OF NASA TIME - E' Ç A'TE \\ (TIMES OF THE EMERALD)
}

${ }^{1 *}$ Edna OROZCO, ${ }^{2}$ Fernando OROZCO, ${ }^{3}$ Eraldo Medeiros COSTA-NETO, ${ }^{4}$ Olga Lucia SANABRIA

${ }^{1}$ Universidad del Cauca, Doctorado en Etnobiología y Estudios Bioculturales. Popayán, Cauca, Colombia; ${ }^{2}$ Universidad Nacional de Colombia, Maestría en Enseñanza de las Ciencias Exactas y Naturales. E-mail: feorca7@gmail.com; ${ }^{3}$ Universidad Estatal de Feira de Santana, Departamento de Biología. E-mail: eraldont@uefs.br; ${ }^{4}$ Universidad del Cauca, Doctorado en Etnobiología y Estudios Bioculturales. E-mail: oldiago@unicauca.edu.co; *Autor para correspondencia: elorozco@unicauca.edu.co

\section{RESUMEN}

Submitted: 08/06/2020; Accepted: 12/07/2020

El presente artículo evidencia la relevancia biocultural de los colibríes y sus significados en la cultura indígena Nasa, localizada en Colombia, con mayor influencia en el Departamento del Cauca. Los colibríes en el Cauca alcanzan un número de 100 especies, muchas de ellas endémicas. En la investigación se evidencia su importancia en los tejidos simbólicos de la cultura Nasa, lo cual se hace relevante para su análisis. Preguntas sobre su conservación y abundancia, llevaron a establecer acercamientos y diálogos con la comunidad Nasa a través de sus sabedores, hombres y mujeres de gran sabiduría y autoridades espirituales, conocedores de su identidad como pueblo indígena al cual pertenecen. De estos diálogos, sobre el papel de los colibríes en sus cosmovisiones, estas aves se observan como uno de los principales componentes de la naturaleza que forman parte de la identidad de esta cultura; ocupando una posición importante dentro de la valoración de los elementos de la naturaleza, incluso por sobre muchos otros componentes de la fauna, como elementos que construyen y forman parte de la identidad de un territorio, percibiéndola como una especie biocultural clave, con funciones importantes en varios dominios culturales como por ejemplo, el mito, el arte, el lenguaje, la ritualidad, la política, la identidad del pueblo Nasa, como también de todos los pueblos pertenecientes a la cordillera de los Andes. Es evidente que los colibríes hacen parte del patrimonio zoocultural de Sudamérica y cumplen un papel clave en las tramas bioculturales de esta región. Cabe resaltar que esta investigación preliminar intenta avanzar hacia un análisis sobre los colibríes en Colombia y su valor biocultural; con lo cual quede clara, la comprensión ética, de los valores ecológicos, culturales y patrimoniales de las aves y la promoción de una conservación integrada de la diversidad biológica y cultural.

Palabras-clave: Colibrí, Especie biocultural clave, diversidad biocultural, Etnoornitología.

\begin{abstract}
This article shows the biocultural relevance of hummingbirds and their meanings in the Nasa indigenous culture, located in Colombia, with the greatest influence in the Department of Cauca. Hummingbirds in Cauca reach a number of 100 species, many of them endemic. The research shows its importance in the symbolic tissues of the Nasa culture, which becomes relevant for its analysis. Questions about its conservation and abundance, led to establish approaches and dialogues with the Nasa community, through its experts, men and women of great wisdom and spiritual authorities who are aware of their identity as indigenous people to which they belong. From these dialogues, about the role of hummingbirds in their worldviews, these birds are seen as one of the main components of nature that are part of the identity of this culture; These birds occupy an important position within the valuation of the elements of nature, even above many other components of fauna, as elements that build and form part of the identity of a territory, perceiving it as a key biocultural species, with functions important in various cultural domains such as myth, art, language, rituality, politics, the identity of the Nasa people, as well as of all the peoples belonging to the Andes mountain range. It is evident that hummingbirds are part of the zoocultural heritage of South America and play a key role in the biocultural plots of this region. It should be noted that this preliminary research tries to advance towards an analysis for hummingbirds and their biocultural value for Colombia, with which it is clear, the ethical understanding of the ecological, cultural and patrimonial values of birds and the promotion of conservation integrated biological and cultural diversity.
\end{abstract}

Key words: Hummingbird, Key biocultural species, Biocultural diversity, Ethnoornithology 


\section{INTRODUCCIÓN}

Las aves se evidencian en el registro etnográfico como un componente de las diversas prácticas cognitivas de las culturas humanas, lo que demuestra que estos animales han sido, históricamente, objetos y símbolos significativos para una amplia gama de sociedades en todo el mundo (Martínez, 2013; Galvagne-Loss et al., 2014; Navarijo Ornelas, 2019), existiendo una sólida relación de los humanos y las aves, seres únicos y fecundos que han mistificado y cautivado por miles de años a la humanidad.

Desde de la Etno-ornitología, se hace posible explorar las formas complejas de relacionamiento humano con el universo avifaunístico, tema fundamental en el siglo XXI, no solo por la significancia de estos organismos en la naturaleza, sino por su papel histórico-cultural en las sociedades humanas. Los etno-ornitólogos afirman que las aves son claramente importantes para los seres humanos y dan como evidencia la gran cantidad de historias sobre aves que superan a la de cualquier otro organismo (Navarijo Ornelas, 1999; Tidemann y Gosler, 2012). En este sentido, es necesario mencionar la emergencia de la etno-ornitología (Tidemann y Gosler, 2012; Vásquez-Dávila y Lope-Alzina, 2012) con una mirada teórica que trata de ir más allá del estudio de las aves en las culturas analizando su relación con el arte, usos, lenguajes, formas de vida, diálogos e interacciones en la vida cotidiana, además de una propuesta metodológica que posibilita un camino de profundización en las relaciones entre aves, humanos y otros seres, con el fin de compatibilizar el conocimiento ecológico indígena y local con el científico en los programas de conservación y gestión del medio ambiente (Tidemann y Gosler, 2012: 9; Galvagne-Loss y Costa Neto, 2017).

Como lo argumenta Bonta (2012: 13), la etno-ornitología tiene un gran potencial para promover la conservación de la biodiversidad. Al principio de disciplinas sociales como la Antropología, las relaciones humanas con los animales y el mundo natural se abordaron en gran medida a través del análisis del totemismo y el mito animista (Malinowski, 1926; Radcliffe-Brown, 1929; Evans-Pritchard, 1956; Douglas, 1957; Levi-Strauss, 1971; Durkheim, 1995; Frazer, 2010). Cuando los antropólogos comenzaron a estudiar las estructuras fundamentales de la cultura considerada primitiva, así como el parentesco, la religión y las creencias cosmológicas, encontraron a las aves como actores principales dentro de las narrativas y prácticas analógicas de la identidad social (Durkheim, 1995).

En la actualidad, la biodiversidad se constituye en un tema que reviste profunda importancia para la sociedad a nivel mundial, el cual es uno de los objetivos de estudio de las ciencias biológicas en todos los tiempos. Tomando un lugar preponderante para las ciencias sociales a finales del siglo XX, ha dado origen a un nuevo campo de la investigación: la Diversidad Biocultural (Harmon y Loh, 2004; Loh y Harmon, 2005; Maffi, 2004, 2005, 2007). El concepto de diversidad biocultural comprende las siguientes premisas: la diversidad de la vida que se compone de especies biológicas, hábitats, ecosistemas y genomas, la diversidad cultural refiriéndose a la variedad de grupos étnicos y la diversidad lingüística por la pluralidad de lenguas (Maffi, 2005; Toledo y Barrrera-Bassol, 2008).

Los enfoques contemporáneos sobre la conservación de la biodiversidad y la cultura han suscitado una serie de análisis, en los cuales se brinda una nueva perspectiva al acercamiento de la relevancia biocultural, mediante la conceptualización de las especies bioculturales clave (Garibaldi y Turner, 2004; Ellen, 2006; Platten y Henfrey, 2009; Ibarra et al., 2012), una adaptación metafórica del concepto ecológico de especie-clave de Paine (1969), definiéndola como aquella que mantiene la estructura y funcionamiento en un ecosistema. Garibaldi y Turner (2004) desarrollaron un concepto análogo y metafórico a partir de la definición de Paine (1969), contextualizando en general a las especies bioculturales clave como aquellas que funcionan como íconos culturales, que tienen un lugar preponderante en la lengua, ceremonias y narraciones, es decir, que forman las bases contextuales de las culturas y, por lo tanto, son propicias para fomentar la conservación y la restauración biocultural.

Desde esta perspectiva, se analiza a los colibríes o picaflores (Aves: Trochilidae) como un complejo de especies con una relevancia biocultural para las culturas originarias de Colombia, con especial énfasis en la cultura de los indígenas Nasa en el Departamento del Cauca, mediante la cual se evidencia la importancia biocultural de estas aves en el tejido simbólico, en la concepción de la vida y el territorio.

Los Trochilidae tienen origen y distribución exclusivamente en el continente americano, es decir, se les encuentra en diferentes ambientes desde Alaska y Canadá hasta la Patagonia. Las mayores concentraciones de especies se localizan en la región tropical, pues suman 165 en Colombia, en Ecuador 163 y en el Perú 118; para México se han contabilizado unas 57 especies de las cuales 24 son endémicas (Navarijo Ornelas, 2019). 
Entre las 100 especies de colibríes que existen en el Cauca, se encuentran tres del género Eriocnemis: E. isabellae, E. mosquera y E. mirabilis (Ayerbe-Quiñones, 2018). Esta última de gran relevancia puesto que al ser descubierta en 1967, promovió a través de sus descubridores una cruzada económica y jurídica para que se decretara el Parque Munchique como Parque Nacional Natural; adicionalmente E. mirabilis y E. isabellae son endémicas del Cauca y en peligro de extinción.

Estas aves se constituyen en seres vivos de gran valor ecológico para los ecosistemas de Colombia, dado que su alta diversidad biológica, ecológica y genética se ve representada en su gran número de especies (165) (Ayerbe-Quiñones, 2018). Colombia es el país con más diversidad en este grupo en toda América, y El Cauca y Nariño son los Departamentos que albergan el mayor número (100 especies cada uno) en Colombia. Esta gran riqueza y el papel ecológico que cumplen a nivel de la polinización, siendo muchas de ellas especies-clave en las redes de interacción colibrí-flor (Ramírez, 2013).

En el mundo Nasa, cultura indígena ubicada al sur-occidente de Colombia y de una profunda influencia en el Departamento del Cauca, los estudios bioculturales son escasos. Sin embargo, al indagar preliminarmente el significado aviar para este pueblo se ha podido observar que las aves son de una gran trascendencia y se asocian con el andar del tiempo en su cosmogonía (el camino del Sol y la Luna), pero además todos los relacionamientos en la vida cotidiana de sus pobladores. Los distintos papeles que juegan las aves en la vida de los Nasa, como compañeros protectores de los humanos y propiciadores de mejores cosechas y fertilidad de sus tierras, o como mensajeros de alegrías y adversidades, de enfermedades y muerte, verifica que para los Nasa las fronteras entre la naturaleza y la cultura se perfilan bajo los supuestos del animismo teorizado por Phillipe Descola (2005), en el cual las especies cuentan con una interioridad, análoga a la de los humanos, y viven en el seno de sociedades complejas que poseen una estructura y propiedades semejantes a las humanas, con jefes, chamanes, rituales, entre otros.

De entre las formas de relación posibles que cita Descola (2005), los indígenas Nasa mantienen relaciones potenciales de intercambio, aprovechamiento, don y conservación con las aves. En este caso, las acciones de dar, tomar e intercambiar cosas entre humanos y otros seres suponen el movimiento de un valor entre dos términos del mismo orden ontológico (Martínez, 2013). En este universo cultural, las aves suelen presentarse como mensajeras y protectoras de los humanos (Descola, 2005), tienen un papel protagónico en los mitos y están sujetas a tabúes sobre varios aspectos cotidianos. Para poder vivir en armonía con los otros seres de la naturaleza, los humanos deben observar y respetar los límites entre ellos y la naturaleza. En la cultura Nasa, al igual que en otros contextos indígenas en América Latina, los humanos y las aves, en lugar de mantener relaciones de antagonismo y poder, dialogan, comparten conocimientos e intercambian puntos de vista (Martínez, 2013).

\section{Los colibríes en la cultura latinoamericana}

Es así, como dentro de este vasto mundo de relaciones humanos-naturaleza, están los colibríes, desde su pequeño tamaño, con una excepcional complejidad natural, observados por miles de años, siendo seres de gran visibilidad en el arte de las culturas en casi todas las sociedades históricas de América (Castro, 2004).

Victoria Castro (2004) ha realizado un importante trabajo investigativo sobre estas aves y sus significados para las poblaciones milenarias de los Andes, el cual se basó fundamentalmente en las clasificaciones y significados. Dentro de algunos relatos citados por Castro (2004), se destacan: en Atacama, los testigos nativos distinguen a Sotar Condi, el picaflor, "como aquel a quien todos los indios de estas Provincias teníamos por Dios teniéndolo nuestro Padre en la mano bestido de cumbe con su pillo [cintillo] y Plumas en él, de oro y Pájaro flamenco [...]” (Castro, 1997). Tratándose, según la autora, así de una deidad regional o pan-regional, que pudo existir desde antes o durante los tiempos del Inka, según algunas evidencias.

Es tal la significancia de los colibríes en América Latina que se observan en trabajos realizados por Arguedas (1981) quien señala que en Puquio, Ayacucho, cada deidad de las montañas de una región poseía su propia ave que lo significaba, donde una de estas, era el quenti o colibrí. Así mismo, Arguedas relata que en el momento en que el sabio religioso, o awki, entró a depositar la ofrenda a las deidades de los cerros dentro de una tumba prehispánica o pukullu, se le apareció un colibrí de vistosos plumajes, que luego desapareció; expresándose de alguna manera la posibilidad de la vida después de la muerte. De esta forma, el colibrí se cualifica desde tiempos coloniales como el "Pájaro que Renace" (Castro, 2004).

Así mismo, la arqueología ofrece buenos ejemplos de la representación del colibrí en la cultura 
Nazca de los Andes costeños del sur del Perú, tanto en la cerámica pintada como en los geoglifos de la Pampa del Ingenio, en donde el pico de esta ave termina en un grupo de líneas que podrían señalar la bajada del Sol el 21 de diciembre. Hoy la gente de Nazca cree que las gigantescas representaciones de aves de este lugar son propiciatorias de la llegada del agua y, tal vez, hace más de mil años los nasquenses las asociaron a la fertilidad de la tierra y la germinación de sus cultivos, beneficiando una producción en un territorio extremadamente árido (Reiche, 1982; Townsend, 1985; Castro, 1990).

En “¿Cómo se percibe en los Andes a sutar, quenti, el colibrí, tomín o picaflor?”, Cobo (1653 [1964]: 323-324) señala lo siguiente:

"El quenti es el menor de los pájaros que se hallan en estas Indias, [...] es tan pequeño, [...] tiene mucha pluma. [...]. Las plumas son pintadas de muchos y hermosos colores. Tiene este pajarito una naturaleza prodigiosa, y es que en las tierras donde se agostan las flores no vive más tiempo de lo que ellas duran, de cuya melosidad se mantiene, sustentándose sobre ellas sin asentarse en el árbol, sino moviendo velozmente sus alitas, se está parado en el aire un buen rato; y en pasándose el tiempo de las flores, se allega a un pino, o a otros árboles, guiado de su natural instinto, y asiéndose con el pico, se queda colgado por tiempo de seis meses, poco más o menos, y en comenzando por la primavera a florecer las plantas, torna él a cobrar vida o despertar de aquel largo sueño".

El texto de Cobo continúa con:

"He oído decir que las damas chilenas adelantan la resurrección de los quentis o Picaflores de su tierra, abrigándolos en su seno. Por eso, y sin necesidad de metáforas místicas, los llaman allí Pájaros resucitados. [...]. Hállanse algunas diferencias dellos, que sólo se distinguen en el tamaño y en los colores de sus plumas. En lengua del Perú se llama este pajarillo, quenti, y en la mexicana, huitzitzil".

Los colibríes comprenden todo un sentido de fertilidad. Se dice de ellos que desaparecen en invierno para retornar en primavera, haciendo referencia a un fenómeno de letargo estacional durante el invierno austral. Considerados por algunos autores como embajadores divinos de esta estación, en que se abren los capullos y empiezan a germinar las plantas. También en la actualidad, en los Andes orientales, los indígenas creen que en los colibríes habitan las almas de los niños muertos que, a veces, se comunican con los vivos (Becker-Donner, no datado). Es así con esta creencia cobra sentido cuando se observa que la gran mayoría de las vasijas Nazca con colibríes pintados provienen de tumbas (Castro, 2004).

Se tiende a considerarlos como la transmigración del bien, por su relación con las flores, y tal vez se trate de una antigua concepción que se encuentra aún hoy en algunas tribus selváticas, donde se piensa que las almas de los muertos premiados se convierten en ellos, gozando para siempre de un "coito eterno" (Castro, 2004). Aparece también en algunos mitos de la región de Huarochirí como comida especial para el halcón, otorgada en premio por su conducta, por uno de los dioses, y en la zona del Cusco es el animal que se encarga de acusar al hombre de todas sus faltas (Cayón, 1971).

En la costa norte del Perú, se concibe actualmente el colibrí como un ave cargada de poder sobrenatural. Debido a su habilidad para succionar, esta ave es asociada con la idea de remover objetos extraños que han sido introducidos por hechiceros en el cuerpo de las personas. No es raro entonces que los médicos kallawaya, de la zona de yungas de Bolivia, reconozcan el poder de sanación que tiene el kenti (colibrí). Actualmente, recomiendan tomar su sangre fresca contra palpitaciones cardíacas y utilizar sus plumas, quemadas y en decocción, contra enfermedades mentales y el susto (Girault, 1987), quedando señalada, etnográficamente, su eficacia simbólica (Castro, 2004).

La mitología mexicana también nombra al colibrí como una de sus deidades, Huitzilopochtli, "Colibrí zurdo" o "Colibrí del sur" (González, 1991: 86). Como parte de los conceptos nahuas de cosmografía y división del tiempo, se visualizaba la presencia de dos colibríes, uno azul turquesa (xuitzil o xiuh-huitzillin) y otro verde (quetzalhuitzil), al inicio de la serie de las 13 aves que rigen las 13 regiones del cielo y las 13 horas del día (Macazaga, 1982 apud Navarijo Ornelas, 2019). En el Códice Magliabecchiano el colibrí aparece con varios de los dioses, entre ellos Quetzalcóatl y el dios del pulque, que llevan como parte de su tocado un punzón de hueso, el símbolo del autosacrificio, teniendo que la sangre que gotea del punzón está simbolizada por una flor o una cadena de plumas y flores, además de un colibrí colgado de la flor usando su pico (Seler, 2004 apud Navarijo Ornelas, 2019).

\section{APORTES METODOLÓGICOS}

El análisis preliminar sobre el valor biocultural del colibrí para la comunidad Nasa, el cual se constituye en el primero realizado en Colombia, ha sido un producto de diálogos realizados entre los 
meses de octubre y diciembre del año 2018 y los meses de marzo y abril del año 2019, con el sabedor Nasa Joaquín Viluche Chocué, hombre de gran sabiduría y estudioso esmerado del camino del conocimiento Nasa, autoridad espiritual del resguardo indígena de Pueblo Nuevo Cauca, quien continuamente señala la necesidad de "dejarse guiar por la naturaleza".

Los diálogos se realizaron durante procesos de Armonización, que es un acto espiritual para buscar la armonía entre quienes la convocan, con todos los seres que los rodean, mediante el sentir de la naturaleza con sus manifestaciones sutiles o evidentes (señas o señales), a través de los mensajeros sabios, que en el caso de la cultura Nasa son los llamados Peysa Yu’çe o Thê'sa We`sx, vínculo entre el hombre y lo sagrado o místico natural. La armonización se obtiene cuando las señales y el sentir están acordes a lo que se llamaría belleza, claridad y fuerza; donde se mambea la hoja sagrada de coca, la cual permite la comunicación con las energías naturales especiales, ya que la hoja de coca tiene su ksxaw o espíritu dador de sabiduría. En el proceso, se pide permiso a los Espíritus de la naturaleza para poder hablar, escribir e investigar, en el caso particular, sobre el colibrí y su relación con la naturaleza y la cultura Nasa. Los diálogos también se llevan a cabo cuando de manera natural se establece una relación amistosa y afectiva con los miembros de la comunidad en conversaciones de trabajo (minga del conocimiento, como trabajo en el TUL o Chagra, espacio de siembra de plantas medicinales y productos de pan-coger al lado de la casa) y vida cotidiana.

El sabedor Joaquín, hombre de 55 años de edad, es docente de la Universidad Autónoma Intercultural Indígena (UAIIN), investigador y autodidacta natural del proceso de conocimiento de su cultura Nasa. Pertenece al Resguardo indígena de Pueblonuevo, cuyo nombre en Nasayuwe (idioma propio del pueblo Nasa) es khwe'nxa Çxhab que significa el pueblo del helecho. Este resguardo se ubica entre los municipios de Caldono y Silvia Cauca, al nororiente del Departamento del Cauca (Colombia). El resguardo hace parte de la Asociación de Cabildos Sa't Tama Kiwe, que significa el Cacique Tama de la Tierra, alusivo a Juan Tama de la Estrella y Calambás, Cacique que unificó y recuperó los títulos coloniales de los cinco pueblos Nasa (Jambaló, Vitoncó, Pitayó, Quichaya y San Francisco de Caldono); Cacique también aceptado por el pueblo Misak.

Importa decir que los datos de este trabajo se obtuvieron mediante el consentimiento informado siguiéndose los lineamientos recomendados por el Código de Ética de la Sociedad Latinoamericana de Etnobiología (Cano-Contreras et al., 2016) y el permiso de la comunidad Nasa, a través de sus sabios espirituales.

En primera instancia se realizaron dos encuentros de armonización. Sin este permiso, sin este vínculo, no es posible recibir de la Madre Tierra los dones esperados, ya que ésta es considerada mujer dadora de vida y requiere de ofrendas especiales expresadas a través del sentir de la "Seña" o "Señales". Es decir, lo que el cuerpo siente, la mente lo piensa, en una unidad indisoluble naturaleza-humano; relación de interpretación armónica que se busca y se encuentra a través de la idea verificada, corregida y potenciada en la misma experiencia, fruto del vínculo directo y milenario con el cosmos, del cual hace parte la Tierra viviente; que se espera el hombre de gran conocimiento (Thê Wala) interprete y armonice sabiamente desde el conocimiento de sus antepasados y su creatividad presente. Todo este tipo de influencias energéticas, que sentidas desde lo cultural se entienden como influencias espirituales o con su propio Espíritu o Ksxaw, solo son posibles si se llevan a cabo sus respectivas armonizaciones y permisos previos al acto mismo de relacionamiento con el ave (Orozco, 2018).

Los pueblos milenarios como los indígenas han mantenido ese vínculo directo con la naturaleza, siendo el pueblo Nasa uno de ellos, es así como se puede evidenciar dicho vínculo en sus prácticas e ideas cotidianas, correspondiendo para este caso particular detallar la relación colibrí-comunidad Nasa.

De estos encuentros donde se hace el mambeo de la planta sagrada E'sx (Coca), y el acompañamiento de los Ksxaw (Espíritus mayores), a través de los Thê Wala (hombres de gran conocimiento), se obtiene el permiso para trabajar en la investigación sobre la importancia del colibrí en la cultura Nasa, por cuanto esta ave es considerada un espíritu fundamental en sus conocimientos de fertilidad de las semillas y abundancia del agua. De estos encuentros surgen varias narrativas que se constituyen en un gran eslabón en el marco conceptual del trabajo de investigación.

\section{VALOR BIOCULTURAL DEL COLIBRÍ - CULTURA NASA}

Para las comunidades Nasa el colibrí es conocido como e'ç (= esmeralda) por sus hermosos colores verdes iridiscentes; esta denominación se extiende por todo el territorio Nasa, que va más allá de su lugar de vivienda; no es únicamente un espacio geográfico, es el lugar donde se hace posible la proyección de la existencia como indígenas, de ahí que se considere como una Yat-Wala (Casa Grande), 
donde se tejen relaciones con los tres espacios que lo integran: el espacio de arriba, el espacio del medio y el espacio de abajo; estas relaciones se ligan a través de prácticas espirituales que acompañan no solo su actuar en la vida cotidiana, sino en la forma como se relacionan con el territorio, al habitarlo, cultivarlo, cuidarlo y defenderlo. Es el lugar donde vivieron los antepasados, donde cultivaron y trabajaron, donde aprendieron y enseñaron a comunicarse con los Espíritus que habitan los lugares sagrados; es el lugar donde se aprenden los saberes integrales que soportan la cultura, constituyéndose en el fundamento central para el desarrollo de la cultura Nasa (SEIP, 2018).

Es en la observación y escucha detallada, junto con el sentir del cuerpo a través de la energía cósmica manifiesta en la Seña, como el pueblo Nasa ha podido aprender a utilizar, respetar y conservar las energías del colibrí. De él comprende que el brillo y color de sus alas llaman luz y lluvia según el tiempo en que se necesite; cuando visita el hogar entiende que habrá visita humana y que su tamaño y forma de aleteo hablarán de si esa visita es alegre, ágil o de sombríos pensamientos o comentarios, de igual forma este aletear y tamaño puede anunciar la presencia del Mayor Trueno o Thê Wala.

Cuando la mujer se encuentra en embarazo es propio frotarle la cabecita del colibrí sobre el vientre y así el niño tendrá los atributos de finura, inteligencia o pensamiento ágil del ave, atributos que de igual manera se pueden obtener si se estrellan dos huevos de colibrí en el cráneo del niño recién nacido (Mayora Filomena Yocue, com. personal, 2019). Si se desea que el niño hombre tenga sus genitales y en especial sus testículos de forma elegante y armónica se le consigue un nido del ave, colocándolo y frotándolo sobre estos órganos, ya que el nido de colibrí es uno de los más hermosos en su redondez y trenzado. De hecho, Navarijo Ornelas (2019) comenta que el colibrí es un símbolo del órgano genital masculino y, en consecuencia, lo es también de la erección y de la virilidad. Si el niño es muy glotón se le da a tomar un café o alguna bebida de su gusto, removida con el pico del colibrí, el cual por su tamaño consume poco alimento.

El colibrí es de tal trascendencia en la naturaleza y de esta con las poblaciones que tienen la sensibilidad de observar y vivenciar su influjo armónico, que ha logrado regular y relacionar determinadas prácticas culturales a lo largo del andar del tiempo. Así, se evidencia que los antepasados sabios Nasa observaron que en la llamada tercera época natural o verano suave (Mezukwe A'te Wala) de este transcurrir del tiempo, avistaban los colibríes entre la segunda y tercera luna, en actitud de pedir abundancia de luz o rayos de sol. Dicha actitud igual se muestra entre la segunda y tercera luna, pero esta vez, en lo corrido de la denominada segunda época natural correspondiente al invierno fuerte (Nus A'te Wala), para pedir abundancia de lluvia necesaria en la siembra del maíz Capio o Kuhtx Wahwa y otras siembras del Tul (espacio doméstico de cultivo de plantas y crianza de animales de la casa).

De esta manera, los sabios antepasados Nasa definieron en la tercera época del transcurrir del tiempo alrededor del sol, el doceavo momento de los veinte existentes de dieciocho días cada uno de su calendario solar, como el momento dedicado al encuentro y armonización con el E'ç (colibrí o esmeralda).

Para la cultura Nasa, en su sabiduría originaria se comparte que todo lo que brilla en el territorio de arriba son estrellas, como el sol, la luna, los planetas quienes están en constante caminar en espiral, por esa razón la Madre Tierra en su giro y su caminar alrededor del sol marca cuatro tiempos, también conocidos como rotación y traslación de la Tierra, la cual produce en el año las cuatro estaciones o las cuatro costillas del tiempo representado en rombo, símbolo de la organización espiritual creado por los Neehwe'sx (protectores o cuidadores del territorio), por lo tanto el camino del tiempo se encuentra dentro de esta Casa Grande (Universo).

Así, para el pueblo Nasa, el tiempo es persona, es ser vivo, es mujer y es hombre, por lo tanto están continuamente caminando en el sentido espiral en los territorios, orientando el tiempo de descanso, de trabajo, de siembra, de no siembra, el tiempo de concentración, de desorientación, el tiempo de paz, de guerra, el tiempo de armonía, de desarmonía. La luna, el sol, las estrellas, Venus y los demás planetas son orientadores de los seres vivos en el universo, por eso la vida de los seres vivos depende si seguimos esas huellas o desviamos de camino: "Para poder seguir las huellas del tiempo, es importante saber escuchar los consejos del tiempo" (Viluche Chocué 2016).

El sol sale cuatro veces en tres sitios diferentes en los filos de las montañas, pudiendo distinguir dos tiempos grandes de aguacero y dos tiempos grandes de sol durante el año; estos tiempos son los guías de la cultura Nasa para las prácticas de sus cinco ceremonias tradicionales, de esta manera se notan cuatro periodos, cada uno de ellos se distribuyen alternadamente permitiendo cambios climáticos, que le dan colores a la naturaleza como forma de vestir especial de la tierra.

En el territorio Nasa, desde épocas milenarias, el tiempo se distribuye en 20 épocas, cada una 
de 18 días y cinco días para las ceremonias principales, conformando un año solar de 365 días (Viluche Chocué, 2016). De manera similar, el calendario lunar se diferencia en ocho lunas alrededor de la Tierra. Cada época y cada momento expresa un conocimiento concreto de la naturaleza en relación con el tiempo de la tierra alrededor del sol. Las cuatro vistas del sol en el territorio Nasa son de vital importancia, significando la llegada y reinicio del camino del sol. De esta manera, el año Nasa comienza con el ritual de los Rayos del Sol Naciente (Sek Buy) (Solsticio de verano, 21 de junio) que da inicio a la época del "Tiempo grande de sol y viento" (Ez A'te Wala) (21 de junio a 21 de septiembre). Siendo el momento para que se reciban las energías y a la vez se ofrezcan bebidas, remedios, comidas y productos del trabajo, para que el sol siga caminando fuerte y dando vida a la Madre Tierra. Este inicio del año es comprensible en todo el territorio ABYA YALA puesto que estos países se encuentran en el camino del sol y luna, muy cerca de la línea ecuatorial; estos espacios son guías para comprender el camino del tiempo y entrar nuevamente al ciclo espiral creado por sus dioses (Viluche Chocué, 2016).

La segunda época, "La época grande de lluvia" (Nus A'te Wala), que va desde 21 de septiembre al 21 de diciembre (equinoccio de invierno), es la época en que el día dura igual que la noche. El ritual correspondiente a esta época es el Saakhelu Ku'jnxisa, referente al agua y las semillas, "tiempo de realizar danza de la culebra verde", para dar la bienvenida de la lluvia. Éste ritual convoca la energía del cóndor que por medio de los colibríes la derrama sobre el territorio para que sea fértil, dando alimento y protección a los seres que habitan en él. La culebra, la rana, los sapos, la libélula y el pez son los mensajeros de la lluvia que cuidan los ojos de agua, se relacionan con el arco iris, ventarrones y granizos. De no realizar esta ceremonia, dicen los mayores que no habrá fertilidad en la tierra porque la energía de la lluvia estará brava, entonces puede no llover o llover demasiado (Viluche Chocué, 2016).

La tercera época llamada "Tiempo grande del sol picante" (Mezukwe A'te Wala), va desde el 21 de diciembre al 21 de marzo (solsticio de verano), tiempo en que la luz del día dura más que el tiempo de la noche. Es el punto donde la tierra se encuentra más cerca del sol. El tercer ritual que se lleva a cabo es propio del "Tiempo del maíz capio" (Khutx Wahwa A'te) o "Tiempo de los negritos" (Kuçx Wala) o "Tiempo de Alegría" (Eçx Eçx A'te), que es el tiempo para agradecer a la Madre Tierra por cuidarnos, dándonos su abrigo y calor, igualmente es momento para pedir perdón a la tierra y ofrendar comida, remedio, bebida y danza, para que ella se contente y rejuvenezca. La época de la siembra del maíz constituye la proyección de la riqueza alimentaria del pueblo Nasa, siendo el maíz el oro que se manifiesta a través de los sueños. En esta época se da el nacimiento de los insectos, como cucarrones, luciérnagas y otros, que son los mensajeros del tiempo; igualmente las visitas de las aves como el colibrí, el chamón y las tijeretas, que son las aves que frecuentan en el cambio del tiempo; las visitas o el movimiento de las "cometas" (êewe), sugieren el trabajo de armonización del territorio, la participación en los rituales de refrescamiento del fuego, donde acontece el diálogo con la anciana brava (pezx buta) (Viluche Chocué, 2016).

La cuarta época se refiere al "Tiempo grande de aguacero suave" (Nus A'te Wala). Época grande de lluvia que va desde el 21 de marzo a 21 de junio (equinoccio de verano), período en que la luz del día dura igual con la noche. El ritual correspondiente a esta época se denomina "Armonización del fuego y la piedra" (Ipx Fxizenxi) y ocurre en el 21 de marzo. Para las familias indígenas y la comunidad, es una de las ceremonias del año de obligatorio cumplimiento. Se debe refrescar al fuego y la piedra para guardar armonía, equilibrio y fuerza en el territorio. La candela es la madre que constantemente brinda gestos de calor humano, abriga y da de comer, es energía que genera vida en la familia, porque allí se realiza la primera siembra del Cordón Umbilical (Sxab Wes). La piedra es la que da fuerza y solidez a las siembras de maíz y arracacha (Arracacia xanthorrhiza Bancroft); así mismo, ayuda a afrontar los problemas de la comunidad (Viluche Chocué, 2016). "Según la noche indicada por los chamanes, la armonización y apagada del fogón se realiza durante toda la noche hasta bien temprano del amanecer, donde es encendido el fuego con remedios frescos, como símbolo de renovación del fuego" (Mayores Sabios Comunidad Nasa, Recopilado por Viluche Chocué, 2016).

De acuerdo a las narrativas del sabedor Nasa Joaquín Viluche Chocué, la comprensión del momento 12avo, en la tercera época "Tiempo grande del sol picante" dentro de sus cinco momentos se observa el tiempo de la esmeralda (E'ç A'te) (del 5 al 22 de enero). La esmeralda o colibrí es el dirigente de las aves pequeñas, es el mensajero de la elegancia, agilidad, lucidez y organización espiritual. Históricamente, cada año en tiempo de verano, con viento, se va a limpiar al cóndor, mientras este está comiendo cabeza, costilla y piernas de ganado. La esmeralda llega con todas las aves pequeñas y le limpian las plumas para que el cóndor permanezca tranquilo durante todo el año, allá en las grandes montañas y no venga anunciando daños a los cultivos. 
El colibrí es el aliado del pueblo Nasa. Los Kiwe Thê (hombres de conocimiento) realizan un ritual especial a este guerrero, le ofrecen miel de abejas, chicha dulce de caña, plantas medicinales frescas, música de flauta y tambor. Es el agradecimiento comunitario por ayudar a mantener sana a la Madre Tierra y por la abundancia en las cosechas.

Desde la sabiduría de los mayores, es sabido que el nido del colibrí se puede quemar dentro de las casas para que estas permanezcan calientes y abrigadas. Las niñas deben repartir de forma equitativa la carne de colibrí asado sin excepción de ninguno integrante del hogar; si la niña realiza este singular trabajo con todos los presentes, entonces, logra esa virtud para los trabajos en la comunidad. Cuando el colibrí visita la vivienda, si esta es ave pequeña avisa que llegarán personas ágiles, amables y trabajadoras, pero si es grande, chilla y llega al patio de la casa es porque el trueno caerá muy cerca de la casa. Del mismo modo, soñar con el colibrí indica que habrá buena suerte y armonía para toda la familia. En estos días se aprende la construcción de casas elegantes y se distingue el ropaje brillante de las aves.

\section{CONCLUSIÓN}

Como se puede evidenciar, los colibríes son aves de profunda importancia para la cultura Nasa, y todas las culturas de América, con un gran simbolismo, desde la cosmovisión y los conocimientos de los pueblos originarios. Para el pueblo Nasa, se evidencian como especies bioculturales clave, teniendo un lugar preponderante en la lengua, ceremonias y narraciones, formando parte de las bases contextuales de su cultura y, por lo tanto, constituyéndose en organismos de relevancia para el fomento de la conservación y la restauración biocultural en los territorios del Departamento del Cauca y en Colombia en general.

El colibrí se observa en la cosmovisión del pueblo Nasa como un gran espíritu que se ha extendido a través de la oralidad y que hoy en día se refleja en la permanencia cultural de sus ceremonias espirituales, en la concepción de su calendario solar y lunar, espíritu que regula y relaciona determinadas prácticas culturales a lo largo del andar del tiempo. El colibrí es un espíritu guardián, regulador de energías de la naturaleza, un aliado del pueblo Nasa, a quien se le ofrenda para pedir abundancia en la reproducción de las semillas y en la abundancia del agua y así, se viva en total armonía, en la Casa Grande o Universo.

\section{LITERATURA CITADA}

Arguedas, J. M. Puquios: una cultura en proceso de cambio. Formación de una cultura nacional indoamericana. México: Siglo XXI editores, 1981.

Ayerbe-Quiñones, F. 2018. Colibríes de Colombia. 2. ed. Washington: Wildlife Conservation Society, 2018.

Bonta, M. Ethno-ornithology and biological conservation. En: Tidemann, S. C.; Gosler, A. (eds.). Ethno-ornithology: birds, indigenous peoples, culture and society. Londres: Earthscan Publications, 2012. p. 13-31.

Cano-Contreras, E.; Medinaceli, A.; Sanabria, O.; Argueta, A. Código de Ética para la investigación, la investigaciónacción y la colaboración etnocientífica en América Latina. Etnobiología, v. 14, supl. L, p. 22-27, 2016.

Castro, V. Artifices del barro. Santiago: Museo Chileno de Arte Precolombiano, 1990.

Castro, V. Huacca Muchay: evangelización y religión andina en Charcas Atacama La Baja. Tesis de Magíster, Universid de Chile, Santiago, 1997.

Castro, V. El picaflor de la gente (Sotar Condi). Ornitología Neotropical, v. 15 (Suppl.), p. 409-417, 2004.

Cayon, E. El hombre y los animales en la cultura quechua. Allpanchis (Cusco), v. 3, p. 135-162, 1971.

Cobo, Fr. B. Historia del Nuevo Mundo. Biblioteca de Autores Españoles, Tomos I, III y IV. Madrid: Atlas, 1653 (1964). Descola, P. Par-delà nature et culture. Paris: Gallimard, 2005.

Douglas, M. Animals in Lele religious symbolism. Africa: Journal of the International African Institute, v. 27, n. 1, p. 46-58, 1957.

Durkheim, É. The elementary forms of religious life. Nueva York: Simon \& Schuster, 1995.

Ellen, R. F. Local knowledge and management of sago palm (Metroxylon sagu Rottboell) diversity in South Central Seram,

Maluku, Eastern Indonesia. Journal of Ethnobiology, v. 26, n. 2, p. 258-298, 2006.

Evans-Pritchard, E. E. Nuer religion. Gloucestershire; Clarendon Press, 1956.

Frazer, J. G. Totemism and exogamy. Londres: Macmillan and Co., 2010.

Galvagne-Loss, A. T.; Costa Neto, E. M.; Machado, C. G; Flores, F. M. Ethnotaxonomy of birds by the inhabitants of Pedra Branca village, Santa Teresinha, municipality, Bahia state, Brazil. Journal of Ethnobiology and Ethnomedicine, 10:55, 2014.

Galvagne-Loss, A. T.; Costa Neto, E. M. Ecología de las aves de acuerdo a los habitantes del poblado de Pedra Branca, Santa Teresinha (Bahía, Noreste de Brasil). Hornero, v. 32, n. 1, p. 73-84, 2017.

Garibaldi, A.; Turner, N. J. Cultural keystone species: implications for ecological conservation and restoration. Ecology

and Society 9:1, 2004.

Girault, L. Kallawaya: curanderos itinerantes de los Andes. La Paz: Unicef, 1987.

Gonzalez, Y. Diccionario de mitología y religión mesoamericana. México: Larousse, 1991. 
Harmon, D.; Loh, J. The IBCD: a measure of the world's biocultural diversity. Policy Matters, v. 13, p. 271-280, 2004.

Ibarra, T. J.; Barreau, A.; Massardo, F.; Rozzi, R. El cóndor andino: una especies biocultural clave del paisaje sudamericano. Boletín Chileno de Ornitología, v. 18, n. 1-2, p. 1-22, 2012.

Levi-Strauss, C. Totemism. 1er. ed. Boston: Beacon Press, 1971.

Loh, J.; Harmon, D. A global index of biocultural diversity. Ecological Indicators, v. 5, p. 231-241, 2005.

Maffi, L. Cultures and conservation: bridging the gap. Policy Matters, v. 13, p. 256-266, 2004.

Maffi, L. Linguistic, cultural and biological diversity. The Annual Review of Anthropology, v. 29, p. 599-617, 2005.

Maffi, L. Biocultural diversity and sustainability. En: Pretty, J. et al. (eds.). SAGE handbook of environment and society. Londres: Sage Publications, 2007. p. 267-277.

Malinowski, B. Myth in primitive psychology. Idaho: Angell Press, 1926.

Martínez, M. Intercambios y diálogos entre aves y humanos. Apuntes etnográficos en la Gunayala de hoy. Canto Rodado, v. 8, p. 41-58, 2013.

Navarijo Ornelas, M. de L. Aves: uso, simbolismo y folklor. México: Instituto de Biología, Universidad Nacional Autónoma de México, 2019.

Navarijo Ornelas, M. de L. Las aves como objetos culturales. En: Vásquez-Dávila, M. A. (ed.). La etnobiología en México: reflexiones y experiencias. Oaxaca: Instituto Tecnológico Agropecuario de Oaxaca SEP; México: Asociación Etnobiológica Mexicana y CONACyT, 1999. p. 87-100.

Orozco-Calambás, F. Elaboración de tejido de conocimiento (Curriculo) para el Programa Universitario Nasa We'sx Jii Fxi'zenxi - con énfasis en investigación del conocimiento de los antepasados y las Ciencias Naturales. Tesis de Maestría, Universidad Nacional de Colombia, 2018.

Paine, R. T. A note on trophic complexity and community stability. American Naturalist, v. 103, p. 91-93, 1969.

Platten, S.; Henfrey, T. The cultural keystone concept: insights from ecological anthropology. Human Ecology, v. 37, n. 4, p. 491-500, 2009.

Radcliffe-Brown, A. R. Notes on totemism in Eastern Australia. The Journal of the Royal Anthropological Institute of Great Britain and Ireland, v. 59, p. 399-415, 1929.

Ramírez, M. Redes de interacción mutualista colibrí-flor en el Parque Nacional Natural Munchique: ¿La pérdida de un colibrí endémico y en peligro crítico de extinción, acarrea el colapso del sistema? Tesis de Maestría, Universidad Nacional de Colombia, 2013.

Reiche, M. Nazca-Perú. Secreto de la pampa. Lauterhofen; Heinrich Fink GmbH, 1982.

Tidemann, S.; Gosler. A. Ethno-ornithology: birds, indigenous peoples, culture and society. Londres: Earthscan Publications, 2012.

Toledo, V.; Barrera-Bassols, N. La memoria biocultural: importancia agroecológica de las sabidurías tradicionales. Barcelona: Icaria editorial, 2008.

Townsend, R. Deciphering the Nazca world: ceramic images from ancient Peru. Art Inst. Chicago Mus. Stud., v. 11, p. 117-139, 1985.

Ulloa, A. Rostros culturales de la fauna: la relación entre los humanos y los animales en el contexto colombiano. Bogotá: Instituto Colombiano de Antropología e Historia, Fundación Natura, 2002.

Vásquez-Dávila, M. A.; Lope-Alzina, D. G. Aves y huertos de México. Oaxaca: Carteles editores, 2012.

Viluche Chocué, J. Nasawe'sx Kiwaka Fxi’zenxi Êen. Popayán: Consejo Regional Indígena del Cauca, 2016. 